\title{
Variable processing of flavors in rat STM
}

\author{
DEREK ROBERTSON and PAUL GARRUD \\ University of St. Andrews, St. Andrews, Scotland
}

\begin{abstract}
Hooded Lister rats exhibited less neophobia towards (i.e., drank more of) a novel fluid (3\% lemon or $5 \%$ sucrose) on a $10-\mathrm{min}$ test if given a 5 -min exposure to that fluid $6 \mathrm{~h}$ earlier. Presentation of a distractor ( $1.25 \%$ coffee) immediately after preexposure to the test solution enhanced neophobia habituation to lemon (Experiment 1), but disrupted habituation to sucrose (Experiment 3). This bidirectional distractor effect was not due to distractor-induced change in the hedonic value of the preexposed test flavor (Experiment 4). Evidence was obtained (Experiment 5) indicating that the rat perceives lemon to be more similar to coffee than is sucrose. It is suggested that when test flavor and distractor are dissimilar, processing of the distractor denies the preexposed test flavor sufficient processing in STM to allow encoding of information about that flavor in LTM. Consequently, the rat responds to a subsequent presentation of the test flavor as it would to a novel stimulus. When test flavor and distractor are similar, however, the distractor elicits less processing in STM (cf. Wagner, 1976) and is therefore less able to disrupt STM processing of the preexposed test flavor. The resultant loss of neophobia to the test flavor resulting from encoding of information about that flavor in LTM may then be augmented by generalization of attenuated neophobia to the distractor. Consistent with this analysis, coffee was shown to suffer more proactive interference when preceded by lemon than when preceded by sucrose (Experiment 6).
\end{abstract}

Exposure of an organism to repeated presentation of a test stimulus results in a diminution of the unconditional response elicited by (i.e., habituation to) that stimulus. If another (distractor) stimulus is experienced after presentation of the test stimulus, however, habituation to the test stimulus is disrupted, that is, "dishabituation" occurs (Pavlov, 1927). Thompson and Spencer (1966) argued that a distractor stimulus did not act to restore responding uniquely to an habituated stimulus, but instead produced a nonspecific increment in responding to all stimuli by increasing the level of excitation or arousal. In other words, purported demonstrations of dishabituation were in fact examples of sensitization. Two studies (Green \& Parker, 1975; Whitlow, 1975) have reported results, however, that cannot readily be explained by invoking a sensitization process.

Green and Parker (1975) demonstrated that habituation of the neophobic response of the rat towards a test solution with which it had had prior experience could be disrupted by presentation of a different nove! fluid during the interval separating preexposure to the test solution and the neophobia test. Dishabituation of the neophobic response to the test solution occurred if the distractor was presented shortly after the preexposure presentation of the test sulution. Contrary to what one might expect if sen-

This research was supported by Grant $B / 80 / 3 / 1203 / 1$ from the Science and Engineering Research Council of Great Britain to the first author. The authors' mailing address is: Department of Psychology, University of St. Andrews, St. Andrews, Fife, Scotland. sitization were responsible for restoration of the neophobic response to the test solution, experiencing the distractor shortly before presentation of the test solution on the neophobia test did not enhance neophobia towards the test solution. In the Whitlow (1975) study, habituation of the unconditional vasoconstriction response of the rabbit to a novel tone was attenuated by interpolation of a visual-tactile stimulus between repeated presentations of the tone. The visual-tactile distractor, however, did not enhance responding to a nonhabituated tone. In other words, the distractor removed a stimulusspecific response decrement without appearing to have a general sensitizing effect. Both the Green and Parker (1975) and the Whitlow (1975) data, therefore, indicate that the mechanism by which a distractor disrupts habituation to a test stimulus may be more complex than that encompassed by the notion of sensitization.

In the experiments reported here we examined further the dishabituating effect of a distractor using a flavor neophobia design similar to that used by Green and Parker. It was found that a distractor need not always disrupt habituation to a test stimulus. Instead, the opposite effect may be obtained, vis., habituation to a test stimulus may be enhanced by presentation of a distractor. Whether a distractor disrupts or enhances habituation to a test stimulus appears to depend on the stimulus characteristics of the distractor and the test stimulus. It is suggested that Wagner's (1976) model of stimulus processing offers a way of explaining the bidirectional effect of a distractor. 


\section{EXPERIMENT 1}

Green and Parker (1975) found that the reluctance of rats to drink a novel flavor solution (i.e., neophobia) was attenuated by a brief exposure to that flavor $6 \mathrm{~h}$ prior to the test of neophobia. Rats that experienced a second novel flavor immediately after preexposure to the test flavor, however, showed an increased reluctance to ingest the test flavor on a subsequent occasion as compared with rats preexposed to the test flavor without a distractor. Green and Parker assumed that consolidation of the memory trace of the preexposed test flavor in long-term memory (LTM) was disrupted by presentation of the distractor. The test flavor was thus more novel (and hence elicited more neophobia) on the subsequent test for rats that had experienced a distractor than it was for rats that had not experienced a distractor following preexposure to the test flavor.

Our original intention was to investigate the merit of an alternative stimulus-generalization-decrement account of the distractor effect obtained by Green and Parker. As a prelude to doing so, it was decided to replicate the Green and Parker (1975) study using flavor solutions different from those used in that study in order to test the generality of the effect. The flavor solutions used in Experiment 1 (i.e., 3\% lemon and $1.25 \%$ coffee) and the relative concentration of each were chosen because they have been commonly used in studies of neophobia and taste aversion learning and were readily available commercially. In addition to the flavor solutions used, Experiment 1 differed from the Green and Parker study in other procedural details, including subjects (Lister vs. Wistar rats), context in which flavors were presented (drinking chamber vs. home cage), and type of test (singlebottle vs. two-bottle presentation).

\section{Method}

Subjects. Twenty-four male Lister rats (165-232 g) were housed in wire-topped plastic cages with dry food pellets continuously available. The room containing the home cages was illuminated on a 12/12-h light/dark cycle (lights on at $0700 \mathrm{~h}$ ).

Apparatus. Testing took place in four open-topped boxes $(25 \times$ $25 \times 40 \mathrm{~cm}$ ) made of black Perspex with a grid floor. A 50 -ml calibrated drinking tube was attached to the outside front wall of the test box by a steel clip, with the nozzle protruding into the box via a hole situated $10 \mathrm{~cm}$ from the side and $6 \mathrm{~cm}$ from the floor of the box. The test boxes were located in a room separate from that in which the home cages were housed.

Procedure. Three days prior to the start of the experiment, access to water in the home cage was restricted to the period 1530 $1600 \mathrm{~h}$. On Experimental Days 1-3, commencing at $1530 \mathrm{~h}$, each rat was placed in the test box for $10 \mathrm{~min}$ and allowed to drink from the calibrated drinking tube. This was intended to habituate rats to the apparatus. Following removal from the text boxes, the rats were returned to the home cage and given 20 -min access to water. The amounts of water consumed in the test box on Days 1-3 were averaged for each rat and divided by its weight. The resulting ratios were rank-ordered and used to assign rats to three groups of eight rats each (i.e., Group PD = preexposed with distractor; Group P = preexposed; Group NP = not preexposed).
Commencing at $0930 \mathrm{~h}$ on Day 4, the test day, Group PD was given a 5 -min presentation of a novel $3 \%(v / v)$ lemon solution followed immediately by a 5 -min presentation of a novel $1.25 \%$ $(w / v)$ coffee solution. Group $P$ received a similar 5 -min presentation of lemon solution followed by presentation of distilled water. Each rat in Group P was matched as closely as possible in terms of weight with a rat in Group PD. The amount of water consumed by rats in Group P was equated with the amount of coffee consumed by the matched-weight controls in Group PD. The third group, Group NP, drank only water during preexposure, the amount of water consumed by each rat in Group NP being equated with the total amount of lemon and coffee solution consumed during preexposure by matched-weight controls in Group PD. Six hours later, the rats were returned to the test box and presented with the $3 \%$ lemon solution. The amount consumed during a $10-$ min period was recorded. A summary of the experimental procedure is provided in Table 1 .

\section{Results and Discussion}

The three groups did not drink equivalent amounts of lemon on the 10-min test (see Table 1). A oneway ANOVA confirmed that these differences were reliable $[F(2,21)=9.88, p<.001]$. Pairwise comparisons using Duncan's multiple range test indicated that Group NP drank significantly less lemon than did either Group P ( $p<.05)$ or Group PD $(p<.01)$. Thus, preexposure to the test flavor attenuated neophobia to that flavor on a subsequent occasion, as reported by Green and Parker (1975). The most interesting outcome of Experiment 1, however, was the performance of Group PD, which drank significantly more lemon than did Group P $(p<.05)$.

The enhanced attenuation of neophobia exhibited by Group PD in Experiment 1 is contrary to the result of the Green and Parker (1975) study, which found that a distractor disrupted attenuation of neophobia to a preexposed novel flavor. It is inconsistent with the view of Green and Parker that a distractor disrupts processing of the test flavor in STM, thereby preventing information about the test flavor gaining access to LTM. Some other explanation of the enhanced habituation of neophobia produced by the distractor in Experiment 1 is thus required.

A number of experimental manipulations have proven effective in attenuating flavor neophobia in rats. For example, handling in infancy (Weinberg, Smotherman, \& Levine, 1978), experience of novel environments (Braveman, 1978), prior presentation of flavors other than the test flavor (Braveman \& Jarvis, 1978; Capretta, Petersik, \& Stewart, 1975; Siegel, 1974), and prior exposure to various odors (Hennessy, Smotherman, \& Levine, 1977) all reduce neophobia to a test flavor. These experiments appear to suggest that prior experience of novel stimulation (regardless of its nature) is sufficient to attenuate flavor neophobia in rats. Accordingly, it was decided to test the hypothesis that Group PD showed less neophobia to the lemon solution than did Group P in Experiment 1 because Group PD drank more novel fluid during the preexposure phase than did Group P. 
Table 1

Design and Results of Experiments 1, 2, 3, and 4

\begin{tabular}{|c|c|c|c|c|c|}
\hline Group & \multicolumn{3}{|c|}{ Stimulus } & \multicolumn{2}{|c|}{ Test Consumption (in milliliters) } \\
\hline \multicolumn{6}{|c|}{ Experiment 1} \\
\hline \multicolumn{6}{|c|}{ Experiment 2} \\
\hline \multicolumn{6}{|c|}{ Experiment 3} \\
\hline $\begin{array}{l}\mathrm{PD} \\
\mathrm{P} \\
\mathrm{NP}\end{array}$ & $\begin{array}{l}\text { sucrose } \\
\text { sucrose } \\
\text { water }\end{array}$ & $\begin{array}{l}\text { coffee } \\
\text { water } \\
\text { water }\end{array}$ & $\begin{array}{l}\text { sucrose } \\
\text { sucrose } \\
\text { sucrose }\end{array}$ & $\begin{array}{r}8.23 \\
11.74 \\
7.70\end{array}$ & $\begin{array}{r}.69 \\
1.17 \\
1.07\end{array}$ \\
\hline \multicolumn{6}{|c|}{ Experiment 4} \\
\hline
\end{tabular}

Note $-P D=$ preexposed with distractor $P=$ preexposed $; N P=$ not preexposed $; G=$ generalization .

\section{EXPERIMENT 2}

If the enhanced attenuation of neophobia observed in Experiment 1 was the result of Group PD's drinking more novel fluid during preexposure than did Group $P$, ensuring equivalent intake of novel flavor solution during the preexposure phase should eliminate this effect. Accordingly, three groups of rats were given a 10-min test of neophobia to the lemon solution. Two of these groups, Groups PD and P, were treated in much the same way as the corresponding groups of the same name in Experiment 1, with the exception that Group $P$ did not receive a water distractor after preexposure to lemon, and the amount of lemon and coffee drunk by Group PD during preexposure was not allowed to exceed the amount of lemon solution drunk by Group P. The third group, Group $G$, was preexposed to an amount of coffee solution equivalent to the amount of lemon drunk by Group $P$ during preexposure. Group $G$ was added to test whether the attenuation of neophobia to lemon observed in Group P depended upon prior exposure to the lemon solution.

\section{Method}

In all unspecified details, the procedure and apparatus in Experiment 2 were identical to those of Experiment 1.

Subjects. The subjects were 18 female Lister rats (116-198 g), housed and maintained in the same way as the rats in Experiment 1.

Procedure. Each rat in Group $P$ was matched with a rat of similar weight in Groups $P D$ and $G$ (where $G=$ generalization). $A$ summary of the experimental procedure is provided in Table 1.

On the test day, rats in Group $P(n=6)$ were given a 5-min presentation of $3 \%(v / v)$ lemon solution. Rats in Group $G(n=6)$ were presented with $1.25 \%(w / v)$ coffee solution, and rats in Group PD $(n=6)$ were presented with the lemon, followed immediately by an equal amount of the coffee solution. The total amount of fluid rats in Groups $G$ and PD were allowed to drink during the preexposure phase was not allowed to exceed the amount of lemon solution drunk by matched-weight control rats in Group P. Six hours later, all rats were given a 10 -min presentation of the lemon solution.

\section{Results and Discussion}

It is clear from Table 1 that the three groups did not differ in the amount of lemon solution they drank on the neophobia test. This was confirmed by a one-way ANOVA $[\mathrm{F}(2,15)=.69, \mathrm{p}>.10]$.

That Group PD did not differ from Group P supports the hypothesis that the enhanced habituation of neophobia towards the lemon solution exhibited by Group PD, relative to that shown by Group P, in Experiment 1 was a result of Group PD's drinking more novel fluid during the preexposure phase than did Group $P$, that is, experience of novelty per se reduces neophobia towards a novel fluid. The comparable amount of lemon ingested by Groups $P$ and $G$ is consistent with this hypothesis, indicating as it does that the strength of neophobia exhibited towards lemon is little influenced by the identity of the solution experienced during the preexposure phase.

Such an explanation of the enhanced habituation effect obtained in Experiment 1 is, however, unsatisfactory in one important respect: it does not explain why rats given a distractor in the Green and Parker (1975) study did not show a similar enhanced attenuation of neophobia given that they also experienced more novel flavor solution during preexposure than did rats preexposed to the test flavor without a distractor. With this in mind, an alternative interpretation of the performance of Group G in Experiment 2 is of interest. The comparable amount of lemon solution ingested by Groups $G$ and $P$ may in- 
dicate that to the rat a $3 \%$ lemon solution is sufficiently like a $1.25 \%$ coffee solution for loss of neophobia to one flavor to generalize to the other. Therefore, Group PD may have shown less neophobia than did Group $P$ in Experiment 1, because loss of neophobia to the distractor generalized to the test flavor and summated with loss of neophobia to the preexposed test flavor itself.

\section{EXPERIMENT 3}

Recently, Wagner (1976) has proposed a model of stimulus processing that appears able to account for both the disruption of neophobia habituation by a distractor that was reported by Green and Parker (1975) and the enhanced habituation of neophobia by a distractor that was obtained in Experiment 1. The model assumes a limited-capacity STM in which stimuli must first be processed before gaining access to LTM. It is further assumed that the amount of processing a stimulus receives in STM depends on its surprise value to the organism: the greater the discrepancy between the contents of STM and information about a stimulus entering in to STM, the more surprising the occurrence of that stimulus will be and hence the more processing it will command.

Both retroactive and proactive interference of STM processing of events are recognized by the model. Because of the limited capacity of STM, the processing commanded by an unexpected event may disrupt processing of immediately preceding events in STM. At the same time, the amount of processing a stimulus commands on entry to STM is subject to proactive interference from items already present in STM. The greater the similarity between an incoming stimulus and the stimuli already present in STM, the less processing the incoming stimulus will attract and the less its ability therefore to retroactively interfere with processing of items already present in STM.

The model predicts, therefore, that a distractor dissimilar to a test flavor will suffer less proactive interference than a distractor that is similar to the test flavor. Consequently, in the former case, the distractor will elicit more processing and hence will be more likely to retroactively disrupt STM processing of the test flavor, thereby preventing information about the test flavor from reaching LTM and disrupting habituation of neophobia to the test flavor.

In contrast, when the distractor and the test flavor are similar, as may have been the case in Experiment 1 , proactive interference from the test flavor will reduce the amount of processing accorded to the distractor, thereby reducing its ability to retroactively disrupt the STM processing necessary for transfer of information about the test flavor to LTM. Loss of neophobia to the test flavor as a result of its encod- ing in LTM might then be augmented by loss of neophobia to the distractor generalizing to the test flavor to produce an enhanced habituation effect like that observed in Experiment 1.

To test the possibility that the direction of a distractor effect is determined by the stimulus characteristics of the test flavor and the distractor, Experiment 3 replicated the conditions of Experiment 1, but with a test flavor assumed to be more dissimilar to the coffee distractor than was the lemon solution used in the previous experiments. Both the lemon and the coffee solutions possessed a strong odor, and while the lemon solution, at least to human senses, did not have any noticeable taste, the coffee tasted bitter. Thus, an odorless, sweet-tasting fluid (5\% sucrose) was chosen as the test flavor in Experiment 3 on the assumption that the rat would perceive it to be less similar to the coffee distractor than was the lemon solution. Group NP, which was not preexposed to sucrose, was expected to drink less sucrose on the neophobia test than did Group P. The comparison of prime interest, however, is that of Group PD with Group P. If the prediction derived from Wagner's stimulus processing model is correct, then one would expect Group PD to drink less sucrose on the neophobia test than Group P.

\section{Method}

In all unspecified details, the procedure and apparatus were identical to those of Experiment 1.

Subjects. The subjects were 20 female Lister rats (133-185 g), housed and maintained in the same way as the rats in the previous experiments.

Procedure. A summary of the experimental procedure is provided in Table 1. On the test day, rats in Group $P(n=7)$ and Group PD $(n=7)$ were given a 5 -min presentation of $5 \%(w / v)$ sucrose solution. This was followed immediately, in the case of Group PD, by a 5 -min presentation of $1.25 \%(w / v)$ coffee solution; rats in Group $P$ were presented with an amount of water equivalent to the amount of coffee ingested by matched-weight control rats in Group PD. Rats in Group NP $(n=6)$ were allowed to drink an amount of water equivalent to the total amount of sucrose and coffee ingested by matched-weight control rats in Group PD. Six hours later, all rats were given a 10 -min presentation of $5 \%$ sucrose solution.

\section{Results and Discussion}

The three groups did not drink comparable amounts of sucrose on the neophobia test (see Table 1). A one-way ANOVA indicated these differences to be reliable $[F(2,17)=4.93, p<.05]$. A posteriori comparisons with Duncan's multiple range test (using a harmonic mean because of unequal $\mathbf{N}$ ) indicated that Groups PD and NP did not differ from one another in the amount of sucrose ingested ( $>.10)$, but both groups drank reliably less sucrose than did Group P $(p<.05)$.

Preexposure to the test solution again attenuated neophobia towards that solution on a subsequent occasion, as is evident from the fact that Group P 
drank more sucrose on the neophobia test than did Group NP. Of more interest, however, is the performance of Group PD, which drank significantly less sucrose than did Group P. In other words, presentation of a distractor disrupted the attenuation of neophobia resulting from preexposure to the sucrose. This result makes it unlikely that the enhanced attenuation of neophobia obtained by use of a distractor in Experiment 1 was the result of Group PD's having drunk more novel fluid during preexposure than did Group P. Instead, the performance of Group PD in Experiment 3 points to stimulus characteristics of the test flavor interacting with those of the distractor to disrupt or enhance attenuation of neophobia towards a preexposed test solution.

\section{EXPERIMENT 4}

The results of Experiment 3 conformed nicely to those expected on the basis of Wagner's (1976) model of stimulus processing. An alternative hypothesis as to the cause of the bidirectional distractor effect observed in Experiments 1 and 3 must, however, be acknowledged: presentation of two solutions in close temporal proximity may produce a symmetrical change in the hedonic value of each solution as a consequence of its association with the other. In terms of the preceding experiments, therefore, if rats initially prefer sucrose to coffee, and coffee to lemon, then the attractiveness of the lemon solution may have been increased and that of the sucrose decreased by virtue of their association with the coffee.

Fortunately, we possessed data from a pilot study in which two groups of rats, Groups NP and P, were tested with a $1.25 \%$ coffee solution. As a preliminary check of the hedonic change hypothesis, Group NP of this pilot study was compared with Group NP of Experiment 1 and Group NP of Experiment 3. If the hedonic change hypothesis was correct, one would expect to find that rats drank less lemon than coffee, and less coffee than sucrose (i.e., lemon $<$ coffee $<$ sucrose). In fact, the mean amounts of the three solutions ingested were: lemon $(n=8)=4.2 \mathrm{ml}$, coffee $(n=8)=4.4 \mathrm{ml}$, and sucrose $(n=6)=7.7 \mathrm{ml}[F(2,19)$ $=9.94, p<.001]$. Duncan's multiple range test indicated that ingestion of sucrose was significantly greater than that of coffee or lemon $(p<.01)$, which did not differ from one another.

Although this method of assessing the hedonic value of lemon, coffee, and sucrose to the rat has certain limitations, the results of this comparison were of sufficient interest to suggest the desirability of an experiment specifically designed to test the hedonic change hypothesis. Accordingly, the conditions of Experiment 3 were replicated using coffee as the test flavor and sucrose as the distractor. If the hedonic change hypothesis is correct, one would expect enhanced habituation of neophobia towards coffee because the coffee solution ought to become more attractive by virtue of its association with the more preferred sucrose solution. In contrast, the Wagner hypothesis would predict disruption of neophobia habituation similar to that observed in Experiment 3, since the order of presentation should not alter the degree to which the coffee and sucrose solution differ from one another (and hence the amount of proactive interference suffered by the distractor on entry to STM and the amount of distractorinduced retroactive interference with processing of the test flavor).

\section{Method}

In all unspecified details, the procedure and apparatus were identical to those of Experiment 1.

Subjects. The subjects were 23 female Lister rats (124-184 g), housed and maintained in the same way as the rats in the previous experiments.

Procedure. A summary of the experimental procedure is provided in Table 1. On the test day, rats in Group $P(n=8)$ and Group PD $(n=8)$ were given a 5 -min presentation of $1.25 \%(w / v)$ coffee solution. This was followed immediately, in the case of Group PD, by a 5 -min presentation of $5 \%(w / v)$ sucrose solution; the rats in Group $P$ were presented with water equivalent in amount to the sucrose consumed by matched-weight control rats in Group PD. Rats in Group NP $(n=7)$ were allowed to drink an amount of water equivalent to the total amount of coffee and sucrose consumed by matched-weight control rats in Group PD. Six hours after the preexposure phase, all rats were given a 10 -min presentation of the coffee solution.

\section{Results}

The amounts of coffee ingested on the 10-min neophobia test are shown in Table 1. It is clear that the three groups did not drink equivalent amounts $[F(2,20)=5.19, p<.05]$. Duncan's multiple range test indicated that Group P drank significantly more coffee than did either Group PD or Group NP $(p<$ $.05)$; the latter two groups did not differ from one another.

As in previous experiments, significant habituation of neophobia occurred as a result of preexposure to the test flavor prior to the neophobia test (Group P vs. Group NP). Of more interest, however, is the performance of Group PD. This group drank less coffee than did Group P and did not differ from Group NP, which had no opportunity to drink coffee prior to the neophobia test. This result is contrary to that predicted by the hedonic change hypothesis, which expected Group PD to drink more coffee than did Group P. The hedonic change hypothesis does not appear, therefore, to be an adequate explanation of the bidirectional distractor effect observed in Experiments 1 and 3 . The disruption by the distractor of attenuated neophobia to the preexposed coffee solution in Experiment 4 was, however, exactly as predicted by the Wagner hypothesis.

\section{Discussion}

A potentially important confound in the preceding experiments that must be addressed is the possibility 
that groups differed in the extent to which they habituated to contextual cues prior to the neophobia test. Although all rats had had equivalent experience of the test box prior to administration of any novel fluid (i.e., $3 \times 10 \mathrm{~min}$ ), matching fluid intake across groups resulted in rats' spending an unequal amount of time in the test box during preexposure to novel solutions. This was so because it took rats less time to drink a fixed amount of water than it took them to drink an equivalent amount of novel solution. Thus, although rats preexposed to a test solution and a novel distractor (Group PD) always spent $10 \mathrm{~min}$ in the test box, rats preexposed to a test solution with a water distractor (Group P) or rats presented with only water during the preexposure phase (Group NP) spent less time in the test box. For the comparison of major concern in these experiments, that is, Group PD versus Group $\mathbf{P}$, this difference was comparatively small; Group $P$, on average, spent from 1 to $1.5 \mathrm{~min}$ less in the test box during the preexposure phase than did Group PD. Taking into account the time spent accustoming rats to drink in the apparatus prior to the administration of novel fluids, the resulting difference between Groups PD and P in terms of total exposure to the contextual cues provided by the test box (40 min vs. from 38 to $38.5 \mathrm{~min}$, respectively) prior to the neophobia test appears insignificant. Nevertheless, the possibility exists that even so small a difference might have been of consequence in determining the strength of neophobia rats exhibited to a novel fluid presented in that context.

Mitchell, Winter, and Moffitt (1980) have demonstrated that habituation to contextual cues may enhance the strength of neophobia exhibited by rats to a novel flavor presented in that context. Thus, the enhanced neophobia towards the test solution exhibited by Group PD, relative to that shown by Group $P$, in Experiments 3 and 4 may be attributable, at least in part, to greater habituation to contextual cues in Group PD. Differential habituation to contextual cues cannot, however, explain the pattern of results obtained in Experiment 1, in which Group PD showed less rather than more neophobia towards the test solution than did Group P, despite the fact that Group PD had greater total experience of the test box than did Group P. If differential context habituation operated upon the strength of neophobia expressed toward a novel fluid in Experiment 1, in the same way as that reported by Mitchell et al. (1980), this would imply that Experiment 1 underestimated the potential for increased attenuation of neophobia in Group PD. Although, therefore, level of habituation to the apparatus may be confounded with preexposure experience of novel fluids in the above experiments, differential context habituation is unable to account for the bidirectional distractor effect observed.

\section{EXPERIMENT 5}

Sucrose was chosen as the test flavor in Experiment 3 because it was assumed to be less similar to the coffee distractor than was the lemon solution used in Experiment 1. Since the explanation offered for the bidirectional distractor effect obtained in Experiments 1 and 3 focuses on assumed differences between the lemon and sucrose solutions in their degree of similarity to the coffee distractor, it was deemed prudent to obtain objective evidence in support of this assumption. Accordingly, one group of rats, Group E, was injected with lithium chloride after drinking coffee solution. Later the rats were offered the choice of drinking either lemon or sucrose solution. If lemon solution is indeed more similar to coffee than is sucrose, one would expect the conditioned taste aversion to coffee to generalize more to lemon than to sucrose. Group E should therefore exhibit a greater preference for sucrose than for lemon. A second group, Group C, was also injected with lithium chloride, but without experiencing the coffee solution. Group $C$ was later offered the same choice of drinking either lemon or sucrose solution. A comparison of Group C with Group E allows a conclusion as to whether any reduced preference for lemon over sucrose exhibited by Group $\mathrm{E}$ is dependent on prior experience of coffee paired with lithium chloride.

\begin{abstract}
Method
Subjects. The subjects were 16 female Lister rats (138-198 g), housed and maintained in the same way as the rats in the previous experiments.

Procedure. A summary of the experimental procedure is provided in Table 2 . One week prior to conditioning, all rats were placed on a $23.5-h /$ day water-deprivation schedule. On the conditioning day, the rats were assigned to one of two groups (each of $n=8$ ). Experimental rats (i.e., Group E) were given a 5-min pre-
\end{abstract}

Table 2

Design and Results of Experiment 5

\begin{tabular}{|c|c|c|c|c|c|c|c|c|c|}
\hline \multirow[b]{3}{*}{ Group } & \multirow{2}{*}{\multicolumn{3}{|c|}{ Procedure }} & \multicolumn{6}{|c|}{ Test Consumption (in milliliters) } \\
\hline & & & & \multicolumn{2}{|c|}{ Lemon } & \multicolumn{2}{|c|}{ Sucrose } & \multicolumn{2}{|c|}{ Coffee } \\
\hline & Conditioning & Test 1 & Test 2 & Mean & SE & Mean & SE & Mean & SE \\
\hline
\end{tabular}

Note-C = control $; E=$ experimental; $-=$ paired. 
sentation of novel $1.25 \%$ (w/v) coffee solution followed immediately by a $15-\mathrm{ml} / \mathrm{kg}$ ip injection of $.15 \mathrm{M}$ lithium chloride (LiCl). Control rats (i.e., Group C) were injected with $\mathrm{LiCl}$ after drinking water equivalent to the mean amount of coffee solution drunk by Group E. Following 2 recovery days, in which the rats were allowed to drink water in the test boxes for $10 \mathrm{~min}$, followed by 20 -min access to water in the home cage, each rat was given a two-bottle 10-min preference test involving 3\% (v/v) lemon solution and $5 \%(w / v)$ sucrose solution. For half the animals in each group, the sucrose was initially presented on the left, and for the remaining animals the sucrose was initially presented on the right. After $5 \mathrm{~min}$, the position of the drinking tubes was reversed. The next day, all rats were given a single-bottle presentation of $1.25 \%$ coffee solution for $10 \mathrm{~min}$. (All fluid presentations commenced at $1530 \mathrm{~h}$.

\section{Results and Discussion}

Group E drank less coffee solution than did Group C (see Table 2). This difference was reliable $[t(14)=$ 4.55, $\mathrm{p}<.001$ ], and is consistent with Group E's having acquired an aversion to the coffee solution as a result of experiencing the coffee paired with $\mathrm{LiCl}$ induced toxicosis during the preexposure phase.

Groups $\mathbf{E}$ and $\mathbf{C}$ did not differ in the absolute amount of lemon and sucrose solution they drank on the preference test $[t(14)=.26]$. Although both groups drank more sucrose than lemon solution (see Table 2), the comparison of major interest is the relative magnitude of preference for lemon expressed by Groups $E$ and $C$ (lemon preference scores were calculated using the formula, $L /(L+S) \times 100$, where $L$ $=$ absolute intake of lemon, and $S=$ absolute intake of sucrose in the two-bottle preference test). Group $\mathrm{E}$ exhibited a smaller preference $(\mathbf{4 . 6 \%}$ ) for lemon than that $(10.3 \%)$ exhibited by Group C. Although small, this difference was reliable $[\mathrm{t}(14)=2.47, \mathrm{p}<.05]$. Thus, the conditioned aversion to coffee generalized more to lemon than to sucrose. This supports the argument advanced earlier that the lemon solution is perceived by the rat to be more similar to the coffee solution than is sucrose.

\section{EXPERIMENT 6}

We have argued that the bidirectional distractor effect obtained in Experiments 1 and 3 is best understood in terms of the Wagner (1976) model of stimulus processing. Specifically, we have suggested that, because lemon is more similar to coffee than is sucrose, the coffee distractor elicits less processing when preceded by lemon than it does when preceded by sucrose. Consequently, the ability of the coffee distractor to retroactively interfere with processing of the test solution is less when that solution is lemon than is the case when the test solution is sucrose. In Experiment 5, it was shown that a 3\% lemon solution is perceived by the rat to be more similar to a $1.25 \%$ coffee solution than is a $5 \%$ sucrose solution. Experiment 6 was designed to test the hypothesis that the coffee suffers more proactive interference from the lemon solution than it does from the sucrose.
If lemon does proactively interfere with processing of coffee more than does sucrose, then rats for whom preexposure to coffee is preceded by presentation of lemon solution (Group LC) should be less able to encode information about the coffee in LTM than are rats for whom preexposure to coffee is preceded by presentation of sucrose (Group SC). Group LC should thus drink less coffee on a subsequent test of neophobia to that flavor than do rats for whom preexposure to coffee is preceded by presentation of water (Group WC), whereas Group SC might be expected not to differ from Group WC in the amount of coffee drunk on the neophobia test.

Change in the hedonic value of the preexposed test flavor as a result of its association with the distractor was earlier rejected as an explanation of the pattern of results obtained in Experiments 1 and 3. It is possible, however, that the hedonic value of the solution presented second during the preexposure phase might undergo change as a result of association with the solution presented first. If that is the case, then, unlike the Wagner model, which expects no difference between Groups SC and WC in the amount of coffee drunk on the neophobia test, the hedonic change hypothesis would predict Group SC to drink more coffee than would Group WC, because the attractiveness of the coffee solution to the former group is enhanced by virtue of its association with the more preferred sucrose solution. Both hypotheses, however, make the same prediction regarding the outcome of a comparison between Groups LC and WC.

\section{Method}

In all unspecified details, the procedure and apparatus were identical to that of Experiment 1.

Subjects. The subjects were 47 female Lister rats (141-198 g), housed and maintained in the same way as the rats in the previous experiments.

Procedure. The rats were assigned to one of six groups: Groups SC, LC, WC, SW, LW, and WW. The two letters in the group designations represent the solutions presented during the preexposure phase (i.e., $C=$ coffee, $L=$ lemon, $S=$ sucrose, $W=$ water) and the order of presentation. All groups contained eight rats, with the exception of Group SW, which contained seven rats. A summary of the experimental procedure is provided in Table 3.

On the test day, the rats were presented with $1.5 \mathrm{ml}$ of $3 \%$ (v/v) lemon (Groups LC and LW), $1.5 \mathrm{ml}$ of $5 \%(w / v)$ sucrose (Groups SC and SW), or $1.5 \mathrm{ml}$ of distilled water (Groups WC and WW). This was immediately followed by presentation of either $2.0 \mathrm{ml}$ of $1.25 \%(w / v)$ coffee (Groups LC, SC, and WC) or $2.0 \mathrm{ml}$ of distilled water (Groups LW, SW, and WW). Six hours after the preexposure phase, all six groups were given a 10 -min presentation of the coffee solution.

\section{Results and Discussion}

The groups did not drink comparable amounts of coffee on the neophobia test (see Table 3). A one-way ANOVA indicated that the differences in amount drunk were reliable $[F(5,41)=9.34, p<.0001]$.

A posteriori comparisons were made using Duncan's multiple range test. As in previous experi- 
Table 3

Design and Results of Experiment 6

\begin{tabular}{|c|c|c|c|c|c|}
\hline Group & \multicolumn{3}{|c|}{ Stimulus } & \multicolumn{2}{|c|}{ Test Consumption (in milliliters) } \\
\hline $\mathrm{SC}$ & sucrose & coffee & coffee & 8.10 & .45 \\
\hline WC & water & coffec & coffee & 8.15 & .33 \\
\hline SW & sucrose & water & coffee & 5.01 & .46 \\
\hline LW & lemon & water & coffee & 7.13 & .35 \\
\hline
\end{tabular}

Note $-C=$ coffee $; L=$ lemon $; S=$ sucrose $;$ and $w=$ water.

ments, preexposure to the test solution attenuated neophobia to that solution on a subsequent test, as is evident from the greater amount of coffee drunk by Group WC than by Group WW $(p<.01)$. This attenuation of neophobia to coffee was largely dependent on prior experience of the coffee solution. Rats preexposed to sucrose solution (i.e., Group SW) drank significantly less coffee on the neophobia test than did Group WC $(p<.01)$ and did not differ in amount drunk from Group WW, which had had no experience of novel flavor solutions prior to testing with coffee. By contrast, the rats preexposed to the lemon solution (i.e., Group LW) drank more coffee than did either Group WW or Group SW (ps $<.01$ ). This provides further evidence that a $3 \%$ lemon solution is perceived by rats to be more similar to a $1.25 \%$ coffee solution than is a $5 \%$ sucrose solution.

The comparisons of major interest, however, are those involving Groups WC, SC, and LC. Although all three groups drank the same amount of coffee solution during preexposure, they did not drink equivalent amounts of coffee on the neophobia test. Groups WC and SC drank comparable amounts of coffee solution, but Group LC drank reliably less than either Group WC or Group SC (ps $<.05)$. The comparable amount of coffee drunk during testing by Groups WC and SC is contrary to the expectation of the hedonic change hypothesis, but consistent with that of the Wagner model, and would appear to indicate that the sucrose was ineffective in preventing information about the preexposed coffee solution from being encoded in LTM. By contrast, preceding the coffee solution by lemon during the preexposure phase appeared to reduce the amount of information about the preexposed coffee flavor that was encoded in LTM. This is evident from the greater degree of neophobia exhibited by Group LC towards the coffee during testing than by Groups WC and SC.

These results confirm the hypothesis that coffee suffers more proactive interference from lemon than from sucrose. In fact, since Group LC did not differ from Group LW in the amount of coffee ingested on the neophobia test, it would appear that, under the conditions of this experiment, the lemon may have completely prevented processing of the preexposed coffee solution in STM. This should not, however, be universally true. The extent to which coffee suffers proactive interference from the lemon might be expected to vary as a function of the relative amounts of lemon and coffee rats are allowed to drink during the preexposure phase.

\section{GENERAL DISCUSSION}

Three recent studies (Green \& Parker, 1975; James \& Wagner, 1980; Whitlow, 1975) have reported that the acquisition of habituation to a preexposed test stimulus is disrupted by presentation of a distractor in close temporal proximity to the test stimulus during preexposure. The data reported here are of note, therefore, in that while able to replicate the disruptive effect of a distractor on the acquisition of habituation reported by other authors (Experiment 3), it was also possible to demonstrate the opposite effect, viz., enhancement of habituation, with the same distractor (Experiment 1). The effect of a distractor on acquisition of habituation is not, therefore, invariant.

Whether one obtains distractor-induced disruption or enhancement of neophobia habituation does not appear to be the result of change In the hedonic value of the test solution brought about by association with the distractor (Experiment 4). Instead, it would appear to be related to the stimulus characteristics of the distractor and the test solution. Evidence was obtained (Experiment 5) that the test solution used in Experiment 1, in which enhanced habituation of neophobia was obtained, was more similar to the distractor than was the test solution used in Experiment 3 , in which disruption of neophobia habituation was obtained.

The enhanced habituation of neophobia observed in Experiment 1, when lemon was the test solution, cannot be attributed, however, solely to loss of neophobia to the distractor generalizing to lemon on the neophobia test. If the distractor was as effective in preventing information about the preexposed test solution gaining access to LTM in Experiment 1 as it appeared to be in Experiment 3, then one would expect that, with only generalized loss of neophobia to 
the distractor involved, Group PD would, at best, drink as much lemon on the neophobia test as did Group P. Instead, Group PD drank reliably more lemon than did Group P. One is thus led to conclude that Group PD experienced some loss of neophobia to the preexposed lemon solution in addition to generalized loss of neophobia to the coffee solution. In other words, the coffee distractor was not as effective in preventing informaton about the preexposed test flavor reaching LTM when that flavor was a lemon solution than was apparently the case when the preexposed flavor was sucrose.

It is argued that the bidirectional distractor effect observed in these experiments can be accounted for by invoking Wagner's (1976) model of stimulus processing. The limited-capacity STM system posited by the model means that the amount of processing a test stimulus receives in STM, and hence the likelihood of informaton about that stimulus reaching LTM, may be reduced by processing of a temporally proximate distractor in STM. The more processing elicited by a distractor, the more it will interfere with processing of a preceding test stimulus.

The amount of processing that a distractor receives is directly related to the size of the discrepancy between information about the distractor entering in to STM at the time of its occurrence and information about the distractor already present in STM. A preexposed stimulus that is similar to the distractor will thus occasion less processing of the distractor than is the case when the preexposed stimulus is dissimilar to the distractor (see Experiment 6). Consequently, the preexposed stimulus will undergo less retroactive interference from the distractor in the former case than in the latter. In addition, when there is a degree of similarity between the preexposed stimulus and the distractor, any habituation to the distractor may generalize to the preexposed stimulus and summate with habituation to that stimulus itself. Thus, enhancement of habituation may be expected when the preexposed stimulus and distractor are similar (as was the case in Experiment 1), whereas disruption of habituation may be expected when the distractor and preexposed stimulus are dissimilar (as was the case in Expe-iment 3).

The main focus of attention in explaining the bidirectional distractor effect obtained in the experiments reported here has been directed toward the relative effectiveness of coffee as a distractor when immediately preceded by lemon or sucrose. The assumption underlying this concentration on the role of the coffee distractor is that water did not differentially affect processing of a preexposed lemon or sucrose solution when presented to rats in Group P. This assumption, however, may be unwarranted.

The bidirectional distractor effect obtained in the above experiments may reflect the differential ef- fectiveness of coffee vis à vis water as a distractor when preceded by lemon or sucrose solution; water may have been a better distractor (i.e., more effectively disrupted STM processing of the preexposed test solution) than was coffee when rats were preexposed to lemon, whereas coffee may have been the better distractor when rats were preexposed to sucrose. Given the evidence of Experiment 6 that the processing one stimulus, B, elicits in STM (and hence its ability to disrupt STM processing of a preceding stimulus, A) is inversely related to the degree of similarity that exists between $A$ and $B$, such an analysis would require rats to perceive distilled water (no odor, no taste) as being more similar to sucrose (no odor, sweet taste) than was coffee (strong odor, bitter taste), while perceiving water as being less similar to lemon (strong odor, no taste) than was coffee. The present study does not permit an assessment of this possibility.

\section{REFERENCES}

Braveman, N. S. Preexposure to feeding-related stimuli reduces neophobia. Animal Learning \& Behavior, 1978, 6, 417-422.

Braveman, N. S., \& Jarvis, P. S. Independence of neophobia and taste aversion learning. Animal Learning \& Behavior, 1978, $6,406-412$.

Capretta, P. J., Petersik, T. T., \& Stewart, D. J. Acceptance of novel flavor is increased after early experiences with diverse tastes. Nature, 1975, 254, 689-691.

Green, K. F., \& PArker, L. A. Gustatory memory: Incubation and interference. Behavioral Biology, 1975, 13, 359-367.

Hennessy, M. B., Smotherman, W. P., \& Levine, S. Early olfactory enrichment enhances later consumption of novel substances. Physiology \& Behavior, 1977, 19, 481-483.

JAMES, J. H., \& WAGNeR, A. R. One-trial overshadowing: Evidence of distributive processing. Journal of Experimental Psychology: Animal Behavior Processes, 1980, 6, 188-205.

Mitchell, D., Winte R, W. \& MoffitT, T. Cross-modality contrast: Exteroceptive context habituation enhances taste neophobia and conditioned taste aversions. Animal Learning \& Behavior, 1980, 8, 524-528.

Pavlov, I. P. Conditioned reflexes. Oxford: Oxford University Press, 1927.

Siegel, S. Flavor preexposure and "learned safety." Journal of Comparative and Physiological Psychology, 1974, 87, 1073-1082.

Thomp8on, R. F., \& Spencer, W. A. Habituation: A model phenomenon for the study of neuronal substrates of behavior. Psychological Review, 1966, 73, 16-43.

Whaner, A. R. Priming in STM: An information processing mechanism for self-generated or retrieval-generated depression of performance. In T. J. Tighe \& R. N. Leaton (Eds.), Habituation: Perspectives from child development, animal behavior, and neurophysiology. Hillsdale, N.J: Erlbaum, 1976.

Weingeng, J., Smotherman, W. S., \& Levine, S. Early handling effects on neophobia and conditioned taste aversions. Physiology \& Behavior, 1978, 20, 589-596.

Whitlow, J. W. Short-term memory in habituation and dishabituation. Journal of Experimental Psychology: Animal Behavior Processes, 1975, 1, 189-206.

(Manuscript received March 30, 1983; revision accepted for publication August 9, 1983.) 